Archives

2| 1988

Varia

\title{
Urbanisation et migration des juifs de Hongrie aux $\mathrm{XIX}^{\mathrm{e}}$ et $\mathrm{XX}^{\mathrm{e}}$ siècles
}

\section{Gyula Zeke}

\section{(e) OpenEdition Journals}

\section{Édition électronique}

URL : http://journals.openedition.org/ccrh/2961

DOI : $10.4000 /$ ccrh.2961

ISSN : $1760-7906$

Éditeur

Centre de recherches historiques - EHESS

Édition imprimée

Date de publication : 15 avril 1988

ISSN : 0990-9141

\section{Référence électronique}

Gyula Zeke, "Urbanisation et migration des juifs de Hongrie aux XIX et Xx siècles ", Les Cahiers du Centre de Recherches Historiques [En ligne], 2 | 1988, mis en ligne le 13 avril 2009, consulté le 20 avril 2019. URL : http://journals.openedition.org/ccrh/2961 ; DOI : 10.4000/ccrh.2961

Ce document a été généré automatiquement le 20 avril 2019.

Article L.111-1 du Code de la propriété intellectuelle. 


\title{
Urbanisation et migration des juifs de Hongrie aux XIX et $X X^{e}$ siècles
}

\author{
Gyula Zeke
}

1 En choisissant d'évoquer les rapports entre migration juive et urbanisation en Hongrie aux $\mathrm{XIX}^{\mathrm{e}}$ et $\mathrm{XX}^{\mathrm{e}}$ siècles, on souhaite attirer l'attention sur un aspect mal connu de la modernisation capitaliste en Hongrie. Pour bien situer le problème, il est indispensable d'en évoquer succinctement le contexte historique.

2 À l'instar des autres États d'Europe centrale et orientale, la Hongrie a vu arriver le XIX siècle, époque des grandes transformations, dans des conditions d'arriération. Dans les États européens plus puissants ou plus prospères, le processus de capitalisation s'est effectué dans le cadre politique d'un État national indépendant. En Hongrie, ce processus est resté du domaine des rêves. La révolution hongroise de 1848, qui se situe sur les franges les plus orientales de la vague révolutionnaire européenne, a réussi à détruire la structure économique et politique féodale; elle en vint ensuite tout naturellement à entreprendre une guerre d'indépendance contre l'Empire des Habsbourg. Après les événements que l'on sait, ce n'est qu'en 1867 qu'un compromis passé avec la Monarchie des Habsbourg permit de créer le cadre politique dans lequel la modernisation capitaliste put prendre son essor plus ou moins librement.

3 S'étonnera-t-on alors de l'existence d'une structure sociale arriérée, de caractère féodal ? $\mathrm{Au}$ début du xIX ${ }^{\mathrm{e}}$ siècle, le corps social comprend: d'importantes masses paysannes qui constituent un état distinct et bien organisé ; une noblesse qui, par son pourcentage élevé $(4,6 \%)$, occupe la deuxième place en Europe, juste après la noblesse polonaise ; enfin une bourgeoisie peu nombreuse, d'ethnie principalement allemande.

4 Aucun signe sérieux ne paraît indiquer qu'une bourgeoisie nationale soit susceptible de se développer à partir de l'élite libérale de la noblesse hongroise. Celle-ci se distingue parmi les noblesses européennes en renonçant en 1848 à ses privilèges par l'abolition du servage et de l'inaliénabilité des biens patrimoniaux - ses terres peuvent désormais être achetées et vendues librement - et en acceptant de contribuer aux charges publiques. D'autre part, elle donne au pays une élite intellectuelle et politique de niveau européen, imprégnée 
d'idées libérales, qui constitue après 1867, dans l'administration et la politique, la classe dirigeante de l'État national libéral. Mais, à quelques rares exceptions près, rien ne lui est plus étranger que l'idée d'une entreprise capitaliste, idée radicalement contraire à ses habitudes, à l'orientation de ses aptitudes et à sa conception du monde. La paysannerie, contrainte de rester pour longtemps encore un état distinct, ne parvient qu'à la fin du XIX e siècle, et pour une faible minorité, au seuil de l'embourgeoisement paysan et y stagnera jusqu'à la fin de la seconde guerre mondiale.

5 L'élément urbain, dont l'existence remonte parfois à six ou sept siècles, ne paraît pas non plus susceptible de donner naissance à une bourgeoise nationale. Non pas tant en raison de son origine, qui d'ailleurs s'estompera complètement au $\mathrm{XIX}^{\mathrm{e}}$ siècle grâce à une assimilation rapide et délibérée, que parce qu'il bénéficie de privilèges politiques et économiques de caractère féodal (statut de citoyen de ville libre, système corporatif). Cette situation crée et conforte des habitudes qui font de l'élément urbain un concurrent conservateur des entreprises capitalistes. Ce n'est pas un hasard si les quelques villes, peu peuplées du reste et dont la population juive au tournant du $\mathrm{xx}^{\mathrm{e}}$ siècle compte moins de cent personnes ou ne dépasse que légèrement ce chiffre par la suite, sont des agglomérations qui possèdent depuis longtemps des privilèges féodaux, de grandes traditions et occupent les principaux territoires où s'étaient établis les Allemands au XIII ${ }^{\mathrm{e}}$ siècle.

6 Les Juifs, qui n'immigrent en masse en Hongrie qu'à la fin du XVIII et au début du XIX siècle, sont, pour plus d'une raison, quasi prédestinés à s'introduire dans ce vide structural. Ils y sont portés par leur affinité accrue par nécessité au cours des siècles de la Diaspora, avec le commerce, les affaires financières et les diverses formes d'entreprise ; ils y sont également poussés par les conditions de l'époque en Hongrie. En effet, grâce aux décrets sur les Juifs pris par Joseph II, et malgré leur annulation à la mort du souverain, leur statut d'exclus de la société commence à disparaître. L'année 1840 marque une étape importante : la Diète hongroise leur permet de créer des usines et d'autres entreprises, à condition qu'elles n'emploient que des Juifs. Elle leur accorde également le droit de circuler librement et de s'installer sur tout le territoire du pays, ainsi que le droit de posséder des terres; cette décision ne faisait d'ailleurs que codifier une pratique répandue du droit coutumier. Après la révolution et la guerre d'indépendance de 1848-1849, auxquelles les Juifs hongrois avaient participé avec enthousiasme, le processus s'interrompit pendant une dizaine d'années. Les Juifs vivant en Hongrie durent attendre 1859 pour retrouver leur droit d'acquérir des biens immobiliers - droit qui leur avait déjà été accordé auparavant par la loi de 1840 - et pour profiter d'autres facilités pour l'exercice d'activités économiques ; quant à l'égalité totale en droits, ils ne l'obtiendront que par la loi d'émancipation promulguée en 1867, année du compromis. Le processus législatif qu'il instaure est souvent en retard de plusieurs décennies par rapport à la situation réelle. Le rôle d'avant-garde joué par les Juifs dans la modernisation économique se met en place dans une structure juridique et politique qui empêche son épanouissement.

7 La mise en place de la grande industrie et du commerce en gros ou du système bancaire et de crédit, réalisée en grande partie par les Juifs, est par définition liée à une forte urbanisation. L'importance de ce processus est soulignée par leur participation - souvent supérieure à $50 \%$, au tout début du $\mathrm{xx}^{\mathrm{e}}$ siècle - à l'intelligentsia professionnelle des avocats, médecins, ingénieurs et, d'une manière générale, par leur position de leaders dans l'embourgeoisement de l'ensemble du corps social. 
Quel est le contexte démographique dans lequel s'exerce ce rôle de modernisation? Quels rapports y-a-t-il entre les caractéristiques de la migration et l'urbanisation juive?

Le premier tableau indique les chiffres bruts de la croissance démographique et son taux par rapport à l'ensemble de la population. Il y a des Juifs en Hongrie au Moyen-Age et au début de la période moderne ; leur nombre ne peut faire l'objet que d'estimations, mais leurs communautés sont presque entièrement détruites par la domination turque et les luttes de libération de la fin du XVII ${ }^{e}$ siècle. Au début du XVIII ${ }^{e}$ siècle, c'est une histoire toute nouvelle qui débute, d'un point de vue démographique. Dans cette première période de la nouvelle immigration, les Juifs arrivent surtout de Bohème et de Moravie ; les proportions ne se sont modifiées au profit des territoires de Galicie et d'autres régions du Nord-Est qu'à la fin du XVIII ${ }^{e}$ siècle. A cette époque-là, les villes ne permettent pas aux Juifs, sauf rares exceptions, de s'établir à l'intérieur de leurs murs. C'est l'aristocratie des grands propriétaires qui, pour des raisons d'ordre économique, établit les Juifs dans ses domaines. Des communes entièrement juives sont ainsi créées et quelques-unes où les Juifs sont majoritaires, subsistent exceptionnellement jusqu'à la fin du XIX ${ }^{e}$ siècle. Cette singularité dans l'agglomération et l'établissement des Juifs par les aristocrates - forme de migration non spontanée - est rapidement reléguée au second plan. La répartition territoriale plus ou moins proportionnelle reste typique jusqu'au bout, avec parfois des rassemblements de population plus importants ici ou là. C'est le cas, pour une période relativement durable, dans les départements du Nord-Est du pays qui se trouvent, à partir de la fin du XVIII ${ }^{e}$ siècle, proches du territoire principalement touché par l'immigration. Nulle trace de volonté de concentration à caractère ethnique. Les recherches menées jusqu'à présent, même si elles ne portent pas sur tous les recensements, ne démontrent aucun rapport essentiel entre les tendances régionales de la migration juive et la composition nationale ou religieuse des territoires en question. 
Tableau $\mathrm{n}^{\circ} 1$. Effectifs de la population juive de Hongrie et pourcentage par rapport à la population globale du pays, de 1735 à 1949.

\begin{tabular}{|c|c|c|}
\hline Aunger & chlteffere absolu & $\%$ \\
\hline 17355 & 12.000 & 0.5 \\
\hline 1787 & 83.0000 & $\mathbb{1}$ \\
\hline 1805 & 130.0000 & 1 \\
\hline 18225 & 190.000 & $\mathbb{1}$. \\
\hline 18840 & 242.000 & 2.2 \\
\hline 1850 & 366.000 & 3.2 \\
\hline 1857 & 407.000 & 3.4 \\
\hline 1869 & 542,000 & 4.0 \\
\hline 18830 & 642,000 & 4.6) \\
\hline 1800 & 7007.500 & 4.7 \\
\hline 1900 & 830.000 & 4.9 \\
\hline 19110 & 909.500 & 5.0 \\
\hline $1920^{*}$ & 473.375 & 5. \\
\hline $1930^{18}$ & $4(44.567$ & 5. \\
\hline $1944: 10^{1:}$ & 4000.760 & 4. \\
\hline $19441^{* 111}$ & 725.000 & 4. \\
\hline $1949^{*}$ & 133.862 & 1.5 \\
\hline
\end{tabular}

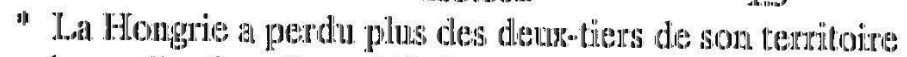
(nowvelles fironatierss deffinies par te Traits de paix de 'Trianon).

"*a" La Hongrie est allors aggrandie des territoines rattachés ajpress les prentiere et dewaibme decisions de Vienne, et de ceur accupes par la suite.

10 Autre caractéristique importante de la croissance de la population juive au XIX ${ }^{\mathrm{e}}$ siècle en Hongrie, croissance dont le rythme exceptionnel se reflète nettement dans le premier tableau : le principal moteur en est l'accroissement naturel, bien supérieur à celui de la population non juive. Faute de données, il est impossible de déterminer avec précision à quel moment cet accroissement naturel devient la source primordiale de la croissance effective, reléguant ainsi au second plan le rôle de l'immigration. Ce tournant a dû se placer relativement tôt, au milieu du siècle au plus tard, car à partir de 1869, l'émigration des Juifs commence à être durablement supérieure à l'immigration; l'accroissement naturel permet donc, comme le tableau l'indique, une augmentation de 370.000 unités de la population juive du pays et compense le départ d'environ 100.000 personnes.

11 Les données de 1920, 1930 et 1941 représentent le nombre des Juifs vivant sur le territoire du pays, tel qu'il a été délimité par le traité de Trianon. Le chiffre de 1949 - date du dernier recensement faisant mention de la confession - reflète le triste bilan de l'Holocauste en Hongrie. Il ressort de ce tableau qu'à l'expansion démographique du siècle dernier succède, entre les deux guerres mondiales, une diminution nette de la population juive de 73.000 personnes. Avant d'en énumérer les raisons possibles, il convient de noter qu'elles sont intimement liées aux traits nouveaux de l'urbanisation de la population juive hongroise, traits qui sont apparus vers la fin du $\mathrm{XIX}^{\mathrm{e}}$ et au début du $\mathrm{XX}^{\mathrm{e}}$ siècle.

12 On ne peut évoquer ici qu'un aspect très limité de l'urbanisation. Si l'on s'en tient à la seule signification des chiffres, on peut laisser dans l'ombre de nombreux paramètres, à une époque où s'édifient la métropole - au sens actuel du terme - et un réseau urbain doté de fonctions radicalement nouvelles. Cette approche n'est pourtant pas sans intérêt en ce qui concerne la population juive hongroise, qui réalise une assimilation d'une 
intensité unique en Europe et des plus réussies en dépit de toutes ses contradictions ultérieures. Son importance est due au fait que le processus d'urbanisation ne coïncide pas seulement avec la transformation fonctionnelle des villes de Hongrie, mais qu'elle en est en fait l'un des principaux facteurs.

Que nous disent les chiffres? Pendant la seconde moitié du XIX ${ }^{e}$ siècle, il n'y a plus une seule ville hongroise où l'on ne trouve des Juifs. A partir de 1840, ils ont l'autorisation légale de s'installer dans les villes, à l'exception des villes dites de mineurs qui leur ferment leurs portes pour vingt ans encore. L'année 1840 ne doit pas être considérée comme une césure. Chaque ville étant libre de laisser ou non libre accès aux Juifs, beaucoup d'entre elles profitent de cette possibilité : en 1825, c'est déjà le cas de la moitié des villes. Les conditions sont changeantes et diverses. Il arrive que les Juifs déjà autorisés à s'installer soient de nouveau expulsés; il existe couramment des permis de séjour valables seulement pour la journée, ainsi que des interdictions concernant l'installation des membres de la famille. Il arrive que les familles juives s'établissent, construisent des maisons en dehors des murs de la ville, puis deviennent citadines au moment de l'extension des limites administratives de la cité.

Rien ne prouve mieux la réussite de cette urbanisation précoce, qui s'effectue dans des conditions juridiques ambivalentes, que la première donnée du tableau $\mathrm{n}^{\circ} 2$. Vers la fin du premier tiers du XIX ${ }^{e}$ siècle, environ un cinquième des Juifs de Hongrie est déjà citadine. Comme le font apparaître les données des années suivantes, le processus s'accélère pendant le dernier tiers du XIX siècle. L'importance de Budapest ne saurait être surestimée. Au tournant du siècle, il n'y a par exemple que 11 villes, à l'exception de Budapest, où le nombre des Juifs soit supérieur à 5000 . Six ans après le compromis, en 1873, la capitale est constituée par la réunion des villes de Pest, Buda et Obuda. Bien que Buda soit l'une des agglomérations juives les plus anciennes de Hongrie, en dépit d'une certaine discontinuité, et qu'Obuda soit l'une des petites communes les plus importantes créées au XVIII ${ }^{e}$ siècle sous l'autorité seigneuriale, l'importance démographique de la capitale est due à la population juive de Pest. Il faut particulièrement souligner l'importance des trois quartiers intérieurs, bâtis à peu près parallèlement à l'immigration. Ces quartiers regroupent près des trois-quarts, puis, entre les deux guerres mondiales, à peu près les deux-tiers de la population juive de la capitale. Notons pour mémoire que, dès 1880 , le nombre des Juifs vivant à Budapest est supérieur à celui de l'ensemble des Juifs vivant en France ou en Angleterre, soit respectivement 60.000 et 65.000 personnes. 
$T^{T a b l e a u ~} n^{\circ} 2$. Effectifs de la population juive citadine de Hongrie ; pourcentage comparatif avec l'ensemble de la population juive de Hongrie, entre 1825 et 1949.

\begin{tabular}{|c|c|c|c|}
\hline Année & $\begin{array}{l}\text { Einsermble du pays } \\
\text { chiffre absolu }\end{array}$ & $\%$ & $\begin{array}{l}\text { Budapest } \\
\text { chiffire absolu }\end{array}$ \\
\hline 1825 & 34.529 & 18.2 & $8.264^{* k * k}$ \\
\hline 1869 & 162.027 & 29.9 & $44.890^{\text {k. } 1 *}$ \\
\hline 1880 & 219.257 & 35.1 & 70.879 \\
\hline 1890 & 274.857 & 38.8 & 103.317 \\
\hline 1900 & 388.060 & 46.8 & 1.68 .985 \\
\hline 1910 & 462.189 & 50.8 & 203.687 \\
\hline $1920^{\text {at }}$ & 344.234 & 72.7 & 215.512 \\
\hline $1930^{: " 1 "}$ & 324.377 & 73.0 & 204.371 \\
\hline $194: 1^{2}$ & 295,398 & 73.7 & 184.453 \\
\hline $1949^{\prime \prime}$ & 107.599 & 80.4 & 96.537 \\
\hline
\end{tabular}

* La Flongrie a perdu plus des deux-tiers de son territoire (nouvelles frontières définies par le Traité de paix de Trianon). * 'Totall des donnés rénaies des villes de Pest, de Buda et d'Obuda.

15 Comme l'indique encore le tableau $\mathrm{n}^{\circ} 2$, le processus spontané de l'urbanisation fait un bond en avant en raison de la réduction du territoire national après la première guerre mondiale. Dans les territoires restés hongrois la défaite militaire, les révolutions, l'occupation roumaine qui y fait suite, la paix de Trianon qui secoue l'ensemble du pays, créent des conditions de choc et d'agitation. Les vagues d'antisémitisme qui déferlent par la suite sur tout le pays font affluer beaucoup de Juifs vers les villes, particulièrement à Budapest où ils sont mieux protégés. Par ailleurs, les Juifs qui fuient les États successeurs, où ils avaient été souvent les principaux agents de la magyarisation, viennent s'ajouter à la colonie juive de Budapest. L'urbanisation qui ne cesse de s'intensifier est cependant, avant tout, le fait d'une migration spontanée. Les causes de ce phénomène nous ramènent au problème de l'importante diminution - en chiffres absolus - à partir de 1920, de la population juive de Hongrie. Trois facteurs principaux sont à considérer.

16 Le premier est la baisse dramatique de l'accroissement naturel. Si celui-ci est au XIX siècle à la base du grand élan de croissance de la population juive, il tend rapidement vers zéro dans les premières décennies $d u x^{e}$ siècle et prend, dès 1927, des valeurs définitivement négatives.

17 L'importance des pertes dues aux conversions n'est pas moindre. Contrairement à la plupart des États d'Europe centrale et orientale et conformément à la conception libérale de l'organisation étatique et de la structure politique depuis 1867, la population juive de Hongrie est considérée comme une communauté confessionnelle. Paradoxalement, les conversions ne commencent à prendre un caractère massif qu'après 1896, c'est à dire au moment où la population juive a déjà obtenu les droits collectifs confessionnels jusqu'alors inexistants et où la religion juive est devenue une religion "reçue", c'est-àdire acceptée. Les mariages mixtes, jusqu'alors réservés aux cas où la partie juive abandonnait sa religion, furent alors possibles et des chrétiens furent libres de se convertir à la religion israélite, ce qui était interdit jusqu'alors. Malgré cela, la conversion qui tantôt fait partie d'une stratégie de défense contre l'antisémitisme, tantôt constitue 
un acte d'assimilation positive, devint l'une des causes principales de la diminution de la population juive. A la fin de cette époque, lourde de menaces, le nombre de ceux qui tout en pratiquant la religion chrétienne sont considérés comme juifs par les lois en vigueur, est d'environ cent mille.

Dernier facteur: l'émigration qui reste importante. Elle s'intensifie encore dans les années 1920 sous l'effet de la loi dite du numerus clausus qui limite l'admission des Juifs aux études supérieures.

Comme pour la population juive des autres pays européens, ces trois facteurs concernent surtout les populations déjà urbanisées. De ce fait, la source exclusive de l'accroissement proportionnel du niveau d'urbanisation est assurée par la migration intérieure. Mais cette prédominance constitue en même temps la cause principale de la diminution globale des effectifs; en effet, la migration ininterrompue, qui caractérise l'ensemble de la période, vers les agglomérations appartenant à un niveau d'urbanisation plus élevé, étroitement liée à une forte mobilité sociale, réduit de plus en plus l'importance de cette partie de la population dont la structure familiale traditionnelle constituait, au XIX siècle, le fondement principal d'un accroissement naturel important. Ainsi, l'urbanisation de la population juive de Hongrie aux $\mathrm{xIX}^{\mathrm{e}}$ et $\mathrm{xx}^{\mathrm{e}}$ siècles constitue-t-elle la principale composante de sa migration. 